\title{
HUMHUB: TAKING SMALL LOCAL TRADE FAIRS ONLINE - COVID-19 CASE STUDY
}

\author{
Luka Skeledzija \\ Student at University of Ljubljana, Ljubljana, Slovenia
}

\begin{abstract}
Due to COVID-19 social distancing measures many events, including small local trade fairs were cancelled in the fall of 2020. By using open-source Facebook-like social networking platform HumHub we were able to organise a small virtual trade fair that attracted over 700 visitors and included 28 virtual booths. By using HumHub we were able to address some key virtual fair platform drawbacks mentioned in the literature. These include ease of use, mobile compatibility, and user familiarity. However, customer retention and ability to measure fair's effectiveness remain problematic. Almost all of the presenters had a positive experience with their virtual booths. It was also confirmed that the motivation for a virtual event in comparison to a physical is about the same for presenters.
\end{abstract}

\section{KEYWORDS}

Virtual Trade Fair, Online Events, e-Commerce, Social Distancing, Open Source

\section{INTRODUCTION}

Trade fairs are a great way for companies in a specific industry to showcase their products and services to new and existing customers, evaluate new channel partners, enhance their brand, assess the latest market trends, and increase sales (Kerin \& Cron, 1987). While big global trade shows are important for big companies, small local trade shows give small business an opportunity to offer their products and services to customers who are closest to them and present their main source of revenue. With the COVID-19 related restrictions in the fall of 2020 in Slovenia, many such shows were cancelled to enforce the necessary social-distancing measures. In the following days, we, the organisers of one of such small trade fairs, decided to take the show online. The rest of this article focuses on how this was achieved and analyses the effectiveness of this approach for trade show organisers, presenters, and attendees.

The advantages of a virtual trade show in comparison to a traditional one according to (Bianchi \& Gottlieb, 2017) are: increased revenue and lower costs, access to distant markets (and an inexpensive way to test new ones), increased brand awareness and credibility, assessment of the market. The disadvantages according to (Bianchi \& Gottlieb, 2017) are: technological problems (programs are difficult to use), inability to measure effectiveness, difficulty in addressing potential customers, and virtual rudeness.

\section{FINDING THE RIGHT PLATFORM}

We helped organise the biggest dog owner's (virtual) festival in Slovenia for the owners of a major local dog and cat blogging websites. A big focus of this project was on pets and products and services that their owners might want or need. During the event we wanted to achieve communication between visitors (as it would naturally happen at a physical event - chat, photos, and video), communication between the visitors and presenters and the ability for presenters and sponsors to showcase their offerings (photos, video, live-streamed video).

A quick review of existing virtual event platforms such as vFair (https:/www.vfairs.com/), Swoogo (https://meet.swoogo.com/) and Inxpo (https://www.inxpo.com/) determined that their pricing is too high for a small local event and that their use was economically unfeasible. Same goes for custom built software. 
Another problem with all these platforms was the complexity and the applications being non-mobile-friendly. Their average customer/visitor is not very tech savvy, so all of this presents new challenges. Turning to the open-source community, an open-source social networking platform HumHub (https://github.com/humhub/humhub) presented itself as the optimal solution to the problem.

"The HumHub platform aims to provide a flexible core platform and some official modules for others to build their own social network with many customization options as a module system, marketplace and theming mechanism as well as an intuitive user interface also suitable for nontechnical users." (cited: HumHub GmbH \& Co. KG, 2020).

In summary, HumHub takes some inspiration from Facebook and open sources it. On HumHub, users can chat with each other, post images, videos, links and embed Youtube Live frames for direct live streaming to the platform. It can also be further customized to fit the brand style, local language and in this way make it a distraction-free local interest-based (online) community space. The central space of the platform is a news feed, where all user and presenter generated content is accessible. HumHub can be self-hosted (no fee) or set up through their service (for a small monthly fee). It is accessible from every modern web browser as a normal website and works very well on mobile devices.

Our first hypothesis was that the presenters and visitors are already very familiar with Facebook, so a similar user interface would help reduce the number of support requests. Secondly, the Facebook-like news feed is optimized for the so-called Users' Time Spent (Bedjaoui, Elouali, \& Benslimane, 2018), so our users will spend on-average more time on this kind of website. Thirdly, we predict that an online fair will be more cost effective than a physical counterpart. Lastly, we predicted that the total amount of the presenters will be lower because of distrust in the success of an online trade fair.

\section{TECHNICAL IMPLEMENTATION AND METHODOLOGY}

In our case HumHub was self-hosted using an AWS md5.large EC2 instance and installed with humhub-docker container provided by mriedmann on Github (https://github.com/mriedmann/humhubdocker). The theme was modified to fit brand style.

To track visitor's actions on the website we used the industry standard Google Analytics website analytics tool (https://analytics.google.com/). To collect feedback from the presenters' view, we interviewed them after the event.

Some demonstrational content was posted to the platform and the URL sent out to potential presenters and advertisers. Each of them was offered a digital booth (Figure 2, Figure 3), from which they could post their content to the public news feed of the website, visible to all registered and non-registered visitors (Figure 1). Visitors could then visit a booth they selected and view all their content. A day before the event all the presenters were given access to the platform to setup their content. The platform opened to the public at $10 \mathrm{AM}$ on the 12th of December 2020. Until midnight the next day, users and presenters were able to interact on the platform, share images, videos, comment, and chat.

Users were driven to the platform by a 5-day Facebook marketing campaign ( 3 days before, 2 days during event) reaching around 100.000 Facebook users. Information about the event was also posted on event organiser's Facebook pages, with approx. 70.000 followers, and on their websites kuzek.si and mucek.si. 


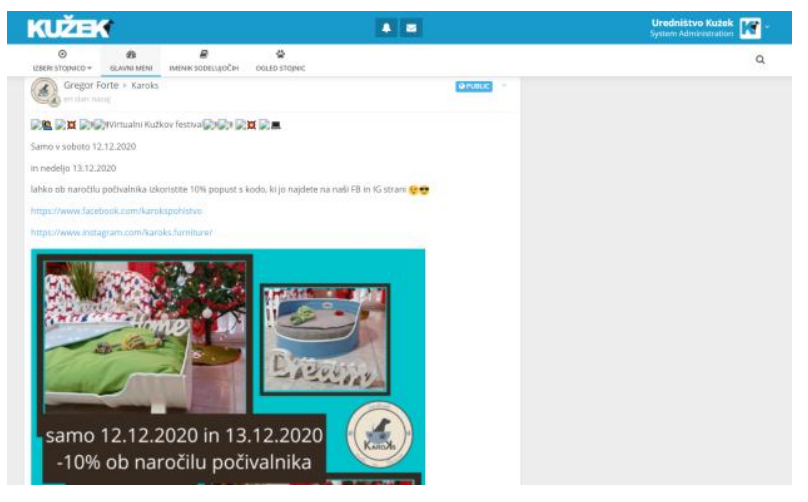

Figure 1. The news feed

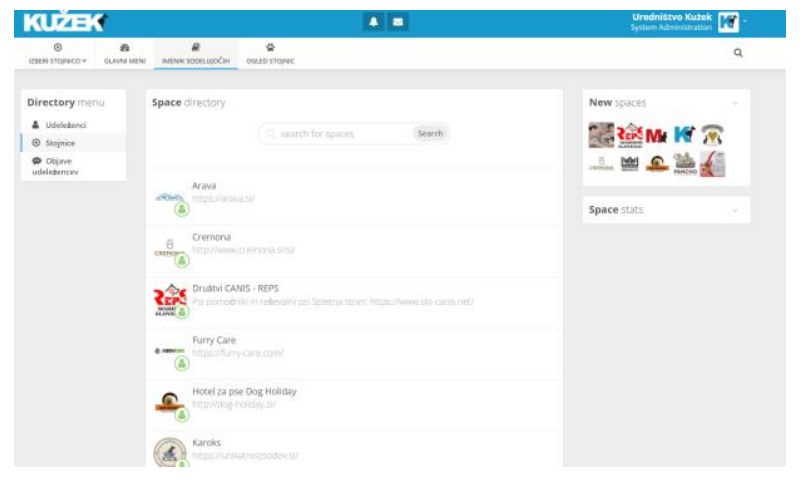

Figure 2. Virtual Booth selection menu

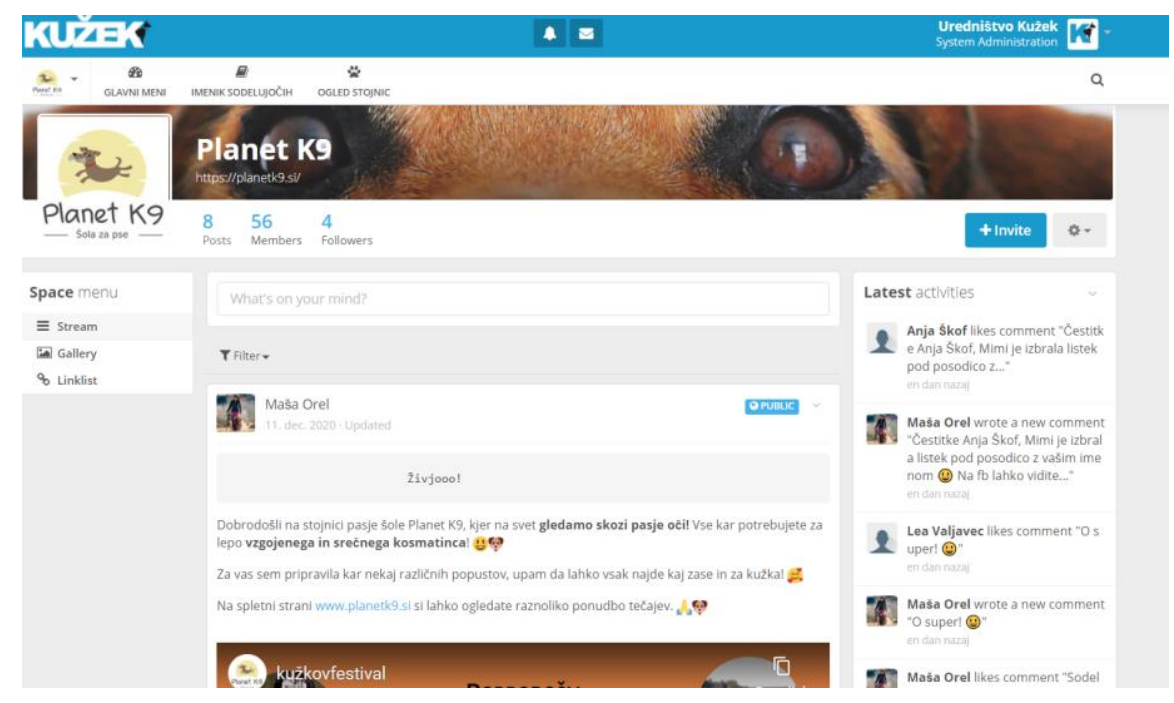

Figure 3. Virtual Booth

\section{ANALYSIS}

In total, there were 28 digital booths active during the event. 11 of them were given to non-profits free of charge, while 17 of them were paid for by advertisers and smaller companies to promote their products and services (available different advertisement packages ranging from 90 to 290 Euros). Prepared materials included videos, images, special discounts, giveaways, success stories regarding their services, dog adoption offers and more.

\subsection{Presenter Experience and Data}

From 30 presenters who had signed up for the physical fair, 28 decided to participate online, which results to customer retention rate of almost $93 \%$ when switching to virtual.

To collect some feedback, we called and interviewed all the presenters. Almost all were happy with their booth package and how their brand was represented. As it turned out, while user interaction on the website was not very high, many people clicked on presenter's links and/or called them to inquire about presented goods and services. 


\subsection{Visitor Behaviour}

According to Google Analytics, during the 2-day period (Saturday and Sunday), 716 unique users attended the digital event with a total of 991 sessions. The number of users who registered on (and not just viewed) the platform is 60 users ( $8 \%$ of all visitors). Consequently, the user engagement in posts made by the presenters was relatively low as well. Most posts got $0-1$ likes, and 0 comments. There were only 2 visitor-generated posts. The most commented on post (a giveaway) got 18 comments.

Although visitor's interaction with the platform was mostly passive, time spent on the platform measured by Google Analytics was very high. In comparison to kuzek.si (their brand name dog blogging website), the time spent on the festival platform (presented under the same brand) was almost 5 times higher.

Collected geoinformation also indicates that the reach of the platform was all over Slovenia. Due to the online nature of the event, we were able to have a presence in regions that are far away from where the physical event would have taken place, increasing the area of brand recognition.

The collected data also indicates (Figure 4) that $74.6 \%$ of people visited the platform from a mobile device, followed by $22.9 \%$ from a desktop computer and $2.6 \%$ from tablet devices. The most active hours were each day from 10 am to 6 pm (Figure 5).

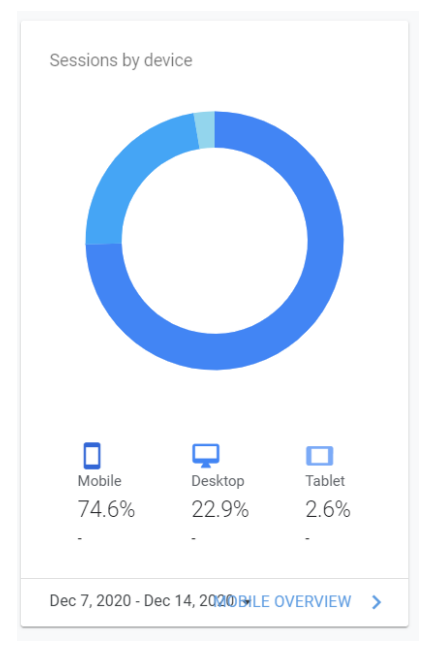

Figure 4. Visitor sessions by device

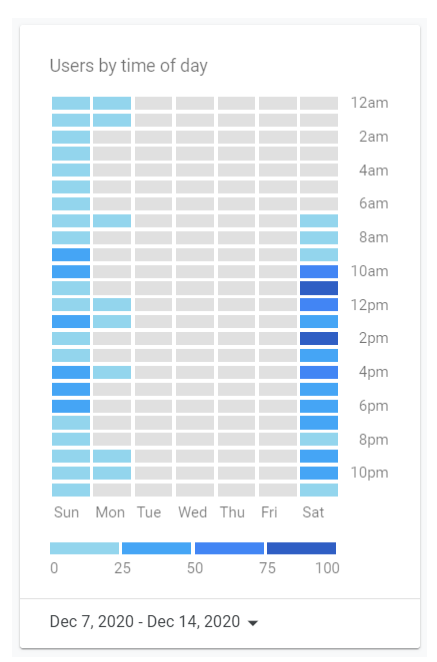

Figure 5. Visitors by time of day

\subsection{Technical Execution Costs}

Our webhosting and bandwidth bill for the week was 16.16 Euros. The use of the platform is free. The setup, customization and technical support required around 24 hours of web developer time. Most of this time was spent customizing the look and behaviour of the platform, so next executions of such events can be cheaper due to the work already done. This greatly decreases the costs in comparison to a few thousand Euros per day for the venue they used to rent.

\subsection{Technical Support Data}

In total, only 2 technical supports requests were made by presenters and 0 by the visitors. The presenters said in the interview that the platform was similar to Facebook, which they know well and intuitively knew how to use. 


\section{CONCLUSION}

In conclusion, this one-time online-based trade fair event proved to be an effective way for companies and advertisers to promote their products in an environment where physical meetups are not possible due to health-related restrictions such as in the case of COVID-19. Advertisers were prepared to pay for digital booths to present their products and services, while also being happy with the representation they got. Most of them were prepared to do it again next year, should the circumstances not change.

It was learned and correctly predicted that the Facebook-like feel of the platform would be very intuitive for both visitors and presenters as indicated by the low number of technical support requests. Secondly, it was learned that Humhub's Dashboard interface is optimized for both Users' Time Spent and mobile devices as indicated by $74 \%$ mobile audience and almost 5 times the amount of time spent on a more traditional blogging site of the same brand. Thirdly, a digital fair was found to be much more cost effective compared to venue rent rates, specially when numerous repetitions of the event are intended. Lastly, it was found that the interest in the digital event was not much lower from presenters' perspective (only 7\% less) as also confirmed by (Bianchi \& Gottlieb, 2017).

Some of the problems, however, remain. Social interaction on the platform was quite low and presenters had no way of approaching visitors should they choose not to contact the presenter themselves. The time spent on the website was high in website perspective, but still very low in comparison to time spent at a physical event. The effectiveness of the event is still hard to measure because feedback collection from users is hard, especially when they interact with the website in a mostly passive manner. This is a problem since customer relationships are the source of value (Geigenmüller, 2010).

A HumHub supported platform turned out to be an effective low-cost alternative for local community events (trade fairs) without a big budget. It works well and could be used in the future while the social-distancing measures last. After this, it still could prove as a cost-effective way of testing and establishing new markets without an actual physical presence. Lastly, it could prove as a very effective marketing method especially for niche product categories where target groups are very geographically dispersed. To conclude, we think the main takeaway of our experiment is that a virtual trade fair doesn't necessarily have to be a complex videogame-like virtual experience. We think it's better to optimize for simplicity and convenience of the users (mobile optimized), which HumHub achieves.

\section{ACKNOWLEDGEMENT}

Our gratitude goes to Mr. Marjan Brecko, the owner of MBMEDIA d.o.o., for giving us access and letting us use the collected analytics data.

\section{REFERENCES}

Bedjaoui, M., Elouali, N., \& Benslimane, S. M. (2018). User Time Spent Between Persuasiveness and Usability of Social Networking Mobile Applications - A Case Study of Facebook and YouTube. Yogyakarta, Indonesia: International Conference on Advances in Mobile Computing \& Multimedia (MoMM2018). doi:10.1145/3282353.3282362

Bianchi, C., \& Gottlieb, U. (2017). Virtual trade shows: Exhibitors' perspectives on virtual marketing capability requirements. Electronic Commerce Research and Applications(21), 17-26. doi:10.1016/j.elerap.2016.12.004

Geigenmüller, A. (2010). The role of virtual trade fairs in relationship value creation. Journal of Business \& Industrial Marketing, 25(4), 284-292. doi:10.1108/08858621011038243

HumHub GmbH \& Co. KG. (2020, December 14). HumHub Documentation. Retrieved from About HumHun: https://docs.humhub.org/docs/about/humhub

Kerin, R. A., \& Cron, W. L. (1987). Assessing Trade Show Functions and Performance: An Exploratory Study. Journal of Marketing, 51(3)(87), 87-94. doi:10.2307/1251649 